\title{
The R-W Metric Has No Constant Curvature When Scalar Factor $R(t)$ Changes with Time
}

\author{
- Influence of Space Curvature on the Densities of Dark Material and Dark Energy
}

\author{
Xiaochun Mei \\ Institute of Innovative Physics in Fuzhou, Department of Physics, Fuzhou University, Fuzhou, China \\ E-mail: ycwlyjs@yeah.net \\ Received September 3, 2011; revised October 26, 2011; accepted November 13, 2011
}

\begin{abstract}
The true meaning of the constant $\kappa$ in the Robertson-Walker metric is discussed when the scalar factor $R(t)$ changes with time. By strict calculation based on the Riemannian geometry, it is proved that the spatial curvature of the R-W metric is $K=\left(\kappa-\dot{R}^{2}\right) / R^{2}$. The result indicates that the R-W metric has no constant curvature when $\dot{R}(t) \neq 0$ and $\kappa$ is not spatial curvature factor. We can only consider $\kappa$ as an adjustable parameter with $\kappa \neq 0$ in general situations. The result is completely different from the current understanding which is based on the precondition that the scalar factor $R(t)$ is fixed. Due to this result, many conclusions in the current cosmology such as the densities of dark material and dark energy should be re-estimated. In this way, we may overcome the current puzzling situation of cosmology thoroughly.
\end{abstract}

Keywords: Cosmology, General Relativity, R-W Metric, Riemannian Geometry, Space-Time Curvature, Dark Material, Dark Energy, Hubble Constant

\section{The R-W Metric has No Constant Curvature when Scalar Factor $R(t)$ is Related to Time}

Standard cosmology takes the Robertson-Walker metric as the basic framework of space-time. According to the principle of cosmology, our universe is uniform and isotropic. It is proved that the space with homogeneity and isotropy is the one with constant curvature [1]. The R-W metric which is considered to be the one with maximum symmetry is

$$
\mathrm{d} S^{2}=c^{2} \mathrm{~d} t^{2}-R^{2}(t)\left(\frac{\mathrm{d} \bar{r}^{2}}{1-\kappa \bar{r}^{2}}+\bar{r}^{2} \mathrm{~d} \theta^{2}+\bar{r}^{2} \sin ^{2} \theta \mathrm{d} \phi^{2}\right)
$$

According to the current understanding, the constant $\kappa$ in (1) is considered to be a spatial curvature factor. That is to say, the R-W metric is with constant curvatures. When space is flat, we have $\kappa=0$ and the metric becomes

$$
\mathrm{d} S^{2}=c^{2} \mathrm{~d} t^{2}-R^{2}(t)\left(\mathrm{d} \bar{r}^{2}+\bar{r}^{2} \mathrm{~d} \theta^{2}+\bar{r}^{2} \sin ^{2} \theta \mathrm{d} \phi^{2}\right)
$$

According to the current understanding, the term of three dimensional spaces in bracket in (2) is flat. By multiplying a scalar factor $R(t)$ which does not depend on spatial coordinates, the spatial term can still be considered as flat. However, this turned out to be not true. We will prove below that when $\dot{R}(t) \neq 0$, the R-W metric has no constant spatial curvature. The spatial curvature is related to $\dot{R}(t)$, although it still does not depend on spatial coordinates.

To explain this result clearly, let's first repeat the deduction procedure of the R-W metric. As we know in geometry that the lower dimensional curved space can be embedded in the higher dimensional flat space. The three dimensional space with a constant curvature $\kappa$ can be considered as a super-curved surface embedded in the four dimensional flat space. The flat space-time metric of four dimensions can be written as

$$
\mathrm{d} S^{2}=\mathrm{d} x_{\mu} \mathrm{d} x_{\mu}=\mathrm{d} x_{i} \mathrm{~d} x_{i}+\left(\mathrm{d} x_{4}\right)^{2}
$$

The condition of three dimensional super spherical surface in the four dimensional space-time is

$$
x_{\mu} x_{\mu}=x_{1}^{2}+x_{2}^{2}+x_{3}^{2}+x_{4}^{2}=\frac{1}{\kappa}=G^{2}
$$


Here $G$ is the radius of super-spherical surface and constant $\kappa$ is the curvature of super-spherical surface. From the formula above, we get

$$
\begin{gathered}
\mathrm{d} x_{4}=-\frac{x_{i} \mathrm{~d} x_{i}}{x_{4}} \\
\left(\mathrm{~d} x_{4}\right)^{2}=\frac{\left(x_{i} \mathrm{~d} x_{i}\right)^{2}}{1 / \kappa-x_{i} x_{i}}
\end{gathered}
$$

Substituting (5) in (3) and introducing coordinate transformation $x_{1}=r \sin \theta \cos \phi, \quad x_{2}=r \sin \theta \sin \phi$, and $x_{3}=r \cos \theta$, we get three dimensional super-curved surface metric with a constant curvature $\kappa$

$$
\mathrm{d} s^{2}=\frac{\mathrm{d} r^{2}}{1-\kappa r^{2}}+r^{2} \mathrm{~d} \theta^{2}+r^{2} \sin ^{2} \theta \mathrm{d} \phi^{2}
$$

When co-moving coordinate $r=R(t) \bar{r}$ is used and time is fixed with $t=t_{0}$ and $R\left(t_{0}\right)=$ constant, (6) can be written as

$$
\begin{aligned}
\mathrm{d} s^{2} & =R^{2}\left(t_{0}\right)\left(\frac{\mathrm{d} \bar{r}^{2}}{1-\kappa R^{2}\left(t_{0}\right) \bar{r}^{2}}+\bar{r}^{2} \mathrm{~d} \theta^{2}+\bar{r}^{2} \sin ^{2} \theta \mathrm{d} \phi^{2}\right) \\
& =R^{2}\left(t_{0}\right)\left(\frac{\mathrm{d} \bar{r}^{2}}{1-\kappa^{\prime} \bar{r}^{2}}+\bar{r}^{2} \mathrm{~d} \theta^{2}+\bar{r}^{2} \sin ^{2} \theta \mathrm{d} \phi^{2}\right)
\end{aligned}
$$

Here $\kappa^{\prime}=\kappa R^{2}\left(t_{0}\right)$ is considered as new space curvature. When time is not fixed and $R(t) \neq$ constant, according the current understanding, we can let $\kappa^{\prime} \rightarrow \kappa$ and extend (7) into

$$
\mathrm{d} s^{2}=R^{2}(t)\left(\frac{\mathrm{d} \bar{r}^{2}}{1-\kappa \bar{r}^{2}}+\bar{r}^{2} \mathrm{~d} \theta^{2}+\bar{r}^{2} \sin ^{2} \theta \mathrm{d} \phi^{2}\right)
$$

On the other hand, the metric of four dimensional space-time in which the three dimensional space has a constant $\kappa$ is

$$
\mathrm{d} S^{2}=c^{2} \mathrm{~d} t^{2}-\left(\frac{\mathrm{d} r^{2}}{1-\kappa r^{2}}+r^{2} \mathrm{~d} \theta^{2}+r^{2} \sin ^{2} \theta \mathrm{d} \phi^{2}\right)
$$

We think that it possible to introduce united cosmic time in the expanding universe with homogeneity and isotropy. So at last, the four dimensional metric of spacetime can be written as

$$
\begin{aligned}
\mathrm{d} S^{2}= & c^{2} \mathrm{~d} t^{2}-\mathrm{d} s^{2}=c^{2} \mathrm{~d} t^{2}-R^{2}(t) \\
& \times\left(\frac{\mathrm{d} \bar{r}^{2}}{1-\kappa \bar{r}^{2}}+\bar{r}^{2} \mathrm{~d} \theta^{2}+\bar{r}^{2} \sin ^{2} \theta \mathrm{d} \phi^{2}\right)
\end{aligned}
$$

This is the standard procedure to derive the R-W metric. It is obvious that the deduction is not strict. We only prove that the metric (6) has a constant curvature $\kappa$. When $R(t) \neq$ constant, we have not proved that the metrics (8) and (9) still have constant curvatures. In fact, when $R(t) \neq$ constant, by using $r=R(t) \bar{r}$ in (6), we get

$$
\begin{aligned}
\mathrm{d} S^{2}= & \frac{\dot{R}^{2}(t) \mathrm{d} t^{2}}{1-\kappa R^{2}(t) \bar{r}^{2}}+\frac{2 \dot{R}(t) R(t) \mathrm{d} t \mathrm{~d} \bar{r}}{1-\kappa R^{2}(t) \bar{r}^{2}} \\
& +R^{2}(t)\left(\frac{\mathrm{d} \bar{r}^{2}}{1-\kappa R^{2}(t) \bar{r}^{2}}+\bar{r}^{2} \mathrm{~d} \theta^{2}+\bar{r}^{2} \sin ^{2} \theta \mathrm{d} \phi^{2}\right)
\end{aligned}
$$

Similarly, substituting $r=R(t) \bar{r}$ in (9), we get

$$
\begin{aligned}
\mathrm{d} S^{2} & =\left[c^{2}-\frac{\dot{R}^{2}(t)}{1-\kappa R^{2}(t) \bar{r}^{2}}\right] \mathrm{d} t^{2}-\frac{2 \dot{R}(t) R(t) \mathrm{d} t \mathrm{~d} \bar{r}}{1-\kappa R^{2}(t) \bar{r}^{2}} \\
& -R^{2}(t)\left(\frac{\mathrm{d} \bar{r}^{2}}{1-\kappa R^{2}(t) \bar{r}^{2}}+\bar{r}^{2} \mathrm{~d} \theta^{2}+\bar{r}^{2} \sin ^{2} \theta \mathrm{d} \phi^{2}\right)
\end{aligned}
$$

We can not get (8) and (10) from Equation (6) and (9). That is to say, when $\dot{R}(t) \neq 0$ or $R(t) \neq$ constant, we have not proved that the constant $\kappa$ is still the spatial curvature factor of the R-W metric!

However, $\kappa$ is generally considered as the curvature constant of the R-W metric and the spatial part of the metric is considered being flat when $\kappa=0$ at present. This is because that the spatial part of three dimension metric in the bracket of (2) is flat. When it multiplies a scalar factor which does not depend on spatial coordinates, the space can be still considered flat. On the other hand, when $R(t) \neq$ constant, we have metric tensor $g_{11} \neq$ constant, (2) is obviously not the metric of flat space. In fact, the metric of the four dimensional flat space-time is

$$
\mathrm{d} S^{2}=c^{2} \mathrm{~d} t^{2}-\left(\mathrm{d} r^{2}+r^{2} \mathrm{~d} \theta^{2}+r^{2} \sin ^{2} \theta \mathrm{d} \phi^{2}\right)
$$

By using co-moving coordinate $r(t)=R(t) \bar{r}$, we get

$$
\begin{aligned}
\mathrm{d} S^{2} & =c^{2}\left[1-\frac{\dot{R}^{2}(t) \bar{r}^{2}}{c^{2}}\right] \mathrm{d} t^{2}-2 R(t) \dot{R}(t) \bar{r} \mathrm{~d} \overline{\mathrm{r}} t \\
& -R^{2}(t)\left(\mathrm{d} \bar{r}^{2}+\bar{r}^{2} \mathrm{~d} \theta^{2}+\bar{r}^{2} \sin ^{2} \theta \mathrm{d} \phi^{2}\right)
\end{aligned}
$$

This form is completely different from the $\mathrm{R}-\mathrm{W}$ metric (2) when $\kappa=0$. The space-time of metric (14) seems to be curved, but it is flat essentially. According to the principle of the Riemannian geometry, if we can find a transformation to turn a curved space into flat, then the original space is flat essentially. If we can not find such a transformation, the original space is a really curved space. Now, because we can find a transformation to turn (14) into (13), the metric (14) is flat essentially. Because we can not find a transformation to turn (2) into (13) when $\dot{R}(t) \neq 0$, the spatial part of (2) can not be flat!

On the other hand, in order to make the spatial distance of the R-W metric be positive, we should have

$$
\frac{R^{2}(t) \mathrm{d} \bar{r}^{2}}{1-\kappa \bar{r}^{2}} \geq 0
$$


So we have $1-\kappa \bar{r}^{2}>0$, i.e., $\kappa<0$ or $0<\kappa<1 / \bar{r}^{2}$. For the observable universe today, we have $\bar{r} \sim 10^{26} \mathrm{~m}$. So for $0<\kappa<1 / \bar{r}^{2}$, we should have $\kappa<10^{-52}$. Therefore, if $\kappa$ is a positive number, it should be very small. If the universe is infinite, we should have $\kappa \rightarrow 0$. In the current cosmology, we take $\kappa=-1$ and 1 as well as 0 to represent the different universes with negative and positive curvatures as well as flat individually. However, $\kappa=1$ is improper to describe the universe, for we have to take $\bar{r}<1$ to ensure the R-W metric meaningful. Unfortunately, this problem is completely neglected in the current theory.

\section{The Spatial Curvature of the R-W Metric}

Now let's precisely calculate the curvatures of (10) under the condition $R(t) \neq$ constant. As we know that curvature has concrete definition in mathematics. We should judge the flatness of space by strict calculation, not by apparent estimation. In the Riemannian geometry, the Riemannian curvature at a certain point of $N$ dimensional space is defined as [2]

$$
K=\frac{R_{\alpha \beta \sigma \rho} p^{\alpha} q^{\beta} p^{\sigma} p^{\rho}}{\left(g_{\alpha \sigma} g_{\beta \rho}-g_{\sigma \beta} g_{\alpha \rho}\right) p^{\alpha} q^{\beta} p^{\sigma} p^{\rho}}
$$

$K$ is related to the selections of direction vectors $p^{\alpha}$ and $q^{\beta}$ at each point in space. We define the covariant tensor $R_{\alpha \beta \sigma \rho}=R_{\alpha \beta \sigma}^{\lambda} g_{\lambda \rho}$ in which $R_{\alpha \beta \sigma}^{\rho}$ and $R_{\alpha \beta \sigma \rho}$ are calculated by following formulas

$$
\begin{aligned}
\Gamma_{\mu \nu}^{\lambda} & =\frac{1}{2} g^{\lambda \alpha}\left(\frac{\partial g_{v \alpha}}{\partial x^{\mu}}+\frac{\partial g_{\alpha \mu}}{\partial x^{\nu}}-\frac{\partial g_{\mu \nu}}{\partial x^{\alpha}}\right) \\
\Gamma_{\alpha \beta, \sigma} & =\Gamma_{\alpha \beta}^{\lambda} g_{\lambda \sigma}=\frac{1}{2}\left(\frac{\partial g_{\beta \sigma}}{\partial x^{\alpha}}+\frac{\partial g_{\sigma \alpha}}{\partial x^{\beta}}-\frac{\partial g_{\alpha \beta}}{\partial x^{\sigma}}\right) \\
R_{\alpha \beta \sigma}^{\rho}= & \frac{\partial}{\partial x^{\beta}} \Gamma_{\sigma \alpha}^{\rho}-\frac{\partial}{\partial x^{\alpha}} \Gamma_{\sigma \beta}^{\rho}+\Gamma_{\lambda \beta}^{\rho} \Gamma_{\sigma \alpha}^{\lambda}-\Gamma_{\lambda \alpha}^{\rho} \Gamma_{\sigma \beta}^{\lambda} \\
R_{\alpha \beta \sigma \rho} & =\frac{1}{2}\left(\frac{\partial^{2} g_{\rho \alpha}}{\partial x^{\sigma} \partial^{\beta}}+\frac{\partial^{2} g_{\sigma \beta}}{\partial x^{\rho} \partial^{\alpha}}-\frac{\partial^{2} g_{\alpha \sigma}}{\partial x^{\rho} \partial^{\beta}}-\frac{\partial^{2} g_{\rho \beta}}{\partial x^{\sigma} \partial^{\alpha}}\right) \\
& +\Gamma_{\rho \alpha, \lambda} \Gamma_{\sigma \beta}^{\lambda}-\Gamma_{\rho \beta, \lambda} \Gamma_{\sigma \alpha}^{\lambda}
\end{aligned}
$$

If curvatures are the same at all points in space, we have $K=$ constant with

$$
K=\frac{R_{\alpha \beta \sigma \rho}}{g_{\alpha \sigma} g_{\beta \rho}-g_{\sigma \beta} g_{\alpha \rho}}
$$

Further, if space is flat, the Riemannian-Christoffel tensors becomes zero everywhere with $R_{\alpha \beta \sigma}^{\rho}=0$ or $R_{\alpha \beta \sigma \rho}=0$.

Now we calculate the space-time curvatures of the R-W metric. The non-zero Christoffel signs are

$$
\begin{array}{ll}
\Gamma_{11}^{0}=\frac{R \dot{R}}{1-\kappa \bar{r}^{2}} \quad \Gamma_{22}^{0}=R \dot{R} \bar{r}^{2} & \Gamma_{33}^{0}=R \dot{R} \bar{r}^{2} \sin ^{2} \theta \\
\Gamma_{11}^{1}=\frac{k \bar{r}}{1-\kappa \bar{r}^{2}} & \Gamma_{01}^{1}=\Gamma_{02}^{2}=\Gamma_{03}^{3}=\frac{\dot{R}}{R} \\
\Gamma_{22}^{1}=-\left(1-\kappa \bar{r}^{2}\right) \bar{r} & \Gamma_{22}^{1}=-\left(1-\kappa \bar{r}^{2}\right) \bar{r} \\
\Gamma_{33}^{1}=-\left(1-\kappa \bar{r}^{2}\right) \bar{r} \sin ^{2} \theta & \Gamma_{12}^{2}=\Gamma_{13}^{3}=\frac{1}{\bar{r}} \\
\Gamma_{33}^{2}=-\sin \theta \cos \theta & \Gamma_{23}^{3}=\operatorname{ctg} \theta
\end{array}
$$

The non-zero curvature tensors of the R-W metric are

$$
\begin{aligned}
& R_{0101}=\frac{R \ddot{R}}{1-\kappa \bar{r}^{2}} \quad R_{0202}=R \ddot{R} \bar{r}^{2} \\
& R_{0303}=R \ddot{R} \bar{r}^{2} \sin ^{2} \theta \quad R_{1212}=\frac{R^{2} \bar{r}^{2}\left(\kappa-\dot{R}^{2}\right)}{1-\kappa \bar{r}^{2}} \\
& R_{1313}=\frac{R^{2} \bar{r}^{2}\left(\kappa-\dot{R}^{2}\right) \sin ^{2} \theta}{1-\kappa \bar{r}^{2}} \\
& R_{2323}=R^{2} \bar{r}^{4}\left(\kappa-\dot{R}^{2}\right) \sin ^{2} \theta
\end{aligned}
$$

The corresponding curvatures are

$$
\begin{aligned}
& K_{01}=K_{02}=K_{03}=-\frac{\ddot{R}}{R} \\
& K_{12}=K_{13}=K_{23}=\frac{\kappa-\dot{R}^{2}}{R^{2}}
\end{aligned}
$$

Here $K_{0 i}$ are the curvature of space-time crossing part, and $K_{k l} \quad(k, l \neq 0)$ are the curvatures of pure space. For $K_{0 i}=0$, we take $\ddot{R}=0$ or $\dot{R}=$ constant. In this case, $K_{k l}=\kappa / R^{2}$ becomes a constant. This is just the result that physicists think of the R-W metric at present. In order to make purely spatial curvature $K_{k l}=0$, we have two chooses. The first is to set $\dot{R}=0$ and $\kappa=0$ simultaneously, representing static space-time. Another is to set $\kappa=-R^{2}\left(t^{\prime}\right)$ at certain moment $t=t^{\prime}$. In this case, we have $K_{k l}=0$ but still have $K_{0 i} \neq 0$. When $\kappa=0$ but $\dot{R}(t) \neq 0$, we have $K_{k l}=-\dot{R}^{2} / R^{2} \neq 0$. The result is completely different from the current understanding.

Now let's estimate the curvature's magnitudes of the R-W metric. Taking $\kappa=0$ in (24), we get

$$
K_{12}=K_{13}=K_{23}=-\frac{\dot{R}^{2}}{R^{2}}
$$

In cosmology, we define the Hubble constant $H(t)=\dot{R}(t) / R(t)$. At present moment $t_{0}$, we have $H\left(t_{0}\right)=H_{0} \sim 2 \times 10^{-18} \mathrm{~s}^{-1}$. So we have $K_{k l} \sim-4 \times 10^{-36}$, i.e., the spatial curvature of the expansive universe is very small. On the other hand, from the equation of cosmology, we have $\ddot{R}\left(t_{0}\right) / R\left(t_{0}\right) \sim 4 \pi G \rho\left(t_{0}\right) / 3 \sim H_{0}^{2} / 2=2 \times 10^{-36} \mathrm{~s}^{-1}$, so we have $K_{0 i} \sim-2 \times 10^{-36}$. The curvature of crossing part of space and tine is also very small. 
Next, how do we recognize the spatial curvature of the metric (2)? In the Riemannian geometry, the intuitive picture is that when a vector moves along a loop and then returns to its starting point, if it can superimpose with original vector, the space is considered flat. If it can not, the space is curved. Therefore, if the space expands with time in the manner of the metric (2), a vector will not superimpose with the original vector when it moves along a loop and returns to the original point. We should recognize the space curvature of the metric (2) in this way.

However, the result of WMAP showed that the space of our universe seems nearly flat $[3,4]$. According to $(24)$, the difference between constant $\kappa$ and $\dot{R}^{2}\left(t_{0}\right)$ should be very mall at present moment. If we think that the constant $\kappa$ has same magnitude with $\dot{R}^{2}\left(t_{0}\right)$, the spatial curvature of the expansive universe may be about $10^{-36}$. As we know that the precision of WMAP is about $10^{-3}$, so the experiment is unable to find such small curvature.

\section{Influence on the Densities of Dark Material and Dark Energy}

The result above may cause great influence on cosmology. By taking into account of cosmic constant $\lambda$, the Friedmann equation of cosmology is

$$
\frac{\dot{R}^{2}}{R^{2}}+\frac{\kappa}{R^{2}}+\lambda=\frac{8 \pi G}{3} \rho
$$

By defining the Hubble constant $H=\dot{R}(t) / R(t)$ and the effective material density of the universe

$$
\begin{aligned}
\rho_{e}(t) & =\rho(t)-3 \lambda /(8 \pi G), \text { we write }(26) \text { as } \\
\rho_{e}(t) & =\frac{3 \dot{R}^{2}(t)}{8 \pi G R^{2}(t)}+\frac{3 \kappa}{8 \pi G R^{2}(t)}=\frac{3 H^{2}(t)}{8 \pi G}+\frac{3 \kappa}{8 \pi G R^{2}(t)}
\end{aligned}
$$

Let $\rho_{e}\left(t_{0}\right)=\rho_{0}, H\left(t_{0}\right)=H_{0}, R\left(t_{0}\right)=1$ and define critical density $\rho_{c}=3 H_{0}^{2} /(8 \pi G)$, (27) becomes

$$
\rho_{0}=\rho_{c}+\frac{3 \kappa}{8 \pi G}
$$

The formula is used to estimate the material density of the universe. If $\kappa$ represents space curvature factor, we have $\kappa=0$ for the flat universe so that the current material density is equal to the critical density. Defining $\Omega=\rho / \rho_{c}$, we have $\Omega_{0}=\rho_{0} / \rho_{c}=1$ for the universe today. However, observations indicate that we only have $\Omega_{0}=0.04$ for normal material, greatly less than 1 . Therefore, non-baryon dark material and dark energy have to be introduced to fill the universe.

However, if $\kappa$ is adjustable parameter, in stead of curvature factor, for the nearly flat universe and practically observed $\rho_{0}$, we can chose proper $\kappa$ to satisfy (28). We take the Hubble constant $H_{0}=65 \mathrm{Km} \cdot \mathrm{s}^{-1} \cdot \mathrm{Mpc}^{-1}$ and obtain $\rho_{c}=7.9 \times 10^{-27} \mathrm{~kg} / \mathrm{m}^{3}$ at present. By practical measurements, we have $\rho_{0} \approx 2 \times 10^{-28} \mathrm{~kg} / \mathrm{m}^{3}$ for luminous material [5-7]. Suppose that practical density of baryon material in the universe is about 10 times more than that of luminous material, i.e., suppose $\rho_{0} \approx 2 \times 10^{-27} \mathrm{~kg} / \mathrm{m}^{3}$, by taking $\kappa=-3.3 \times 10^{-36}$, we can make (28) to be satisfied.

In this way, it becomes unnecessary for us to assume that the non-baryon material is about 6 times more than baryon material if they really exist. As for dark energy, it becomes surplus. We can make theory and observations consistent by adjusting the value of constant $\kappa$ without the hypotheses of dark energy. At last, if $\kappa \neq 0$, from (27) we have

$$
H^{2}(t)=\frac{8 \pi G \rho_{e}(t)}{3}-\frac{\kappa}{R^{2}(t)}
$$

It means that the Hubble constant is not only related to material density, but also relative to scalar factor. At present moment, we have $R\left(t_{0}\right)=1$. According to (28), we have

$$
H_{0}=\sqrt{\frac{8 \pi G \rho_{0}}{3}-\frac{\kappa}{R_{0}^{2}}}=\sqrt{\frac{8 \pi G \rho_{c}}{3}}
$$

Therefore, the Hubble constant is the same.

In fact, if $\kappa$ is an adjustable parameter in stead of a curvature factor, many conclusions of the current cosmology should be re-evaluated. These problems will be discussed later.

\section{Conclusions}

By the strict calculation based on the Riemannian geometry, the paper proves that when the scalar factor $R(t)$ is related to time, the spatial curvature of the R-W metric is $K=\left(\kappa-\dot{R}^{2}\right) / R^{2}$, in stead of $\kappa / R^{2}$. That is to say, the R-W metric has no constant space curvature in general situations. Therefore, if the Friedmann equation is used to describe the expansionary universe, even space is flat, we can only consider $\kappa$ as an adjustable and non-zero parameter. The result may greatly impact cosmology. We need to re-estimate the values of dark material and dark energy densities.

According to the current estimation in cosmology, normal baryon material only makes up $4 \%$, non-baryon dark material makes up $26 \%$ and dark energy makes up $70 \%$ of the universal material. However, nonbaryon dark material with such huge amount of quantity can not been found up to present days, though physicists have struggled to find it for decades.

The situation is even more puzzling for dark energy. In fact, the concept of dark energy is similar to the concept of ether in the nineteen century. Classical physics 
could not explain the light's propagation in the universal vacuum so that the concept of ether as a medium had to be introduced. One purpose that Einstein putted forward special relativity was to eliminate the concept of ether from physics.

Time goes into the twenty-first century, the history is repeating. Because physicists can not explain the high red-shift of supernovae, the concept of dark energy has to be introduced. By the correct understanding of the constant $\kappa$ in the R-W metric and the Friedmann equation, we no longer need the concept of dark energy again. Meanwhile, we may not need to assume that non-baryon dark material is 6 times more than normal baryon material, if non-baryon dark material exists actually. In this way, we may overcome the current puzzling situation of cosmology thoroughly.

\section{Reference}

[1] S. Weinberg, "Gravitation and Cosmology," Science Pub-

\section{Appendix: The Curvatures of Two Dimensional Curved Surfaces in the Three Dimensional Flat Space}

Because the geometrical figure of three dimensional supper spherical surface is abstract and not visualized easily, in order to have a direct visualization, we discuss two dimensional curved surfaces in three dimensional flat spaces further in this section. Similar to (3) and (4), the metric of three-dimensional flat space and the condition of spherical surface are individually

$$
\begin{aligned}
& \mathrm{d} \sigma^{2}=\mathrm{d} x_{1}^{2}+\mathrm{d} x_{2}^{2}+\mathrm{d} x_{3}^{2} \\
& x_{1} x_{1}+x_{2} x_{2}+x_{3} x_{3}=\frac{1}{\kappa}=G^{2}
\end{aligned}
$$

Here constant $\kappa$ is the curvature and $G$ is the radius of two dimensional spherical surface. By using column coordinates $x_{1}=r \sin \theta, \quad x_{2}=r \cos \theta$ and $x_{3}=z$, (31) becomes

$$
\begin{aligned}
& \mathrm{d} \sigma^{2}=\mathrm{d} z^{2}+\mathrm{d} r^{2}+r^{2} \mathrm{~d} \theta^{2} \\
& z^{2}+r^{2}=\frac{1}{\kappa}=G^{2}
\end{aligned}
$$

Here $r=\sqrt{x_{1}^{2}+x_{2}^{2}}$ is the radius of a circle on the plane $z=$ constant. According to the same calculation procedure, we have lishing Company, 1984, p. 441.

[2] S. Zhiming, "Tensor in Physics," Beijing Normal University Publishing Company, Beijing, 1985, p.180.

[3] P. de Bernardis, et al., "A High Spatial Resolution Analysis of the MAXIMA-1 Cosmic Microwave Background Anisotropy Data," Nature, Vol. 404, 2000, pp. 955-959. doi: $10.1038 / 35010035$

[4] C. L. Merriment, et al., "Wilkinson Microwave Anisotropy Probe (WMAP), Observations: Temperature-Polarization Correlation, Astrophysical Journal Supplement Series, Vol. 148, No. 1, 2003, pp. 195-211.

[5] J. H. Oort, "In La Struccture et Evolution de Univers, Institute International de Physique Solvay," R. Stoops, Brussles, 1958, p. 58.

[6] R. G. Carlberg, et al., "Galaxy Cluster Virial Masses and Omega," Astrophysical Journal, 1996, Vol. 462, pp. 3249. doi:10.1086/177125

[7] D. N. Schramm, M. S. Furner, "Big-Bang Nucleosynthesis Enters the Precision Era," Reviews of Modern Physics, Vol. 70, No. 1, 1998, pp. 303-318. doi:10.1103/RevModPhys.70.303

$$
\begin{gathered}
\mathrm{d} z=-\frac{r d r}{\mathrm{z}} \quad \mathrm{d} z^{2}=\frac{(r \mathrm{~d} r)^{2}}{1 / \kappa-r^{2}}=\frac{\kappa(r \mathrm{~d} r)^{2}}{1-\kappa r^{2}} \\
\mathrm{~d} \sigma^{2}=\frac{\mathrm{d} r^{2}}{1-\kappa r^{2}}+r^{2} \mathrm{~d} \theta^{2}
\end{gathered}
$$

The form of (34) is similar to (6). We see that the metric of (32) is flat, but the metric of (34) becomes curved. The reason is that the transformation (33) is non-linear. That is to say, non-linear transformations may change spatial curvatures. Similarly, by introducing nonlinear transformation $r=R(z) \bar{r}$ and let $R^{\prime}(z)=d R(z) / d z$ in (34), we obtain

$$
\begin{aligned}
\mathrm{d} \sigma^{2}= & \frac{R^{\prime 2}(z) \bar{r}^{2} \mathrm{~d} z^{2}}{1-\kappa R^{2}(z) \bar{r}^{2}}+\frac{R^{\prime}(z) R(z) \bar{r} \mathrm{~d} z \mathrm{~d} \bar{r}}{1-\kappa R^{2}(z) \bar{r}^{2}} \\
& +R^{2}(z)\left[\frac{\mathrm{d} \bar{r}^{2}}{1-\kappa R^{2}(z) \bar{r}^{2}}+\bar{r}^{2} \mathrm{~d} \theta^{2}\right]
\end{aligned}
$$

Let

$$
\mathrm{d} S^{2}=\mathrm{d} z^{2}+\mathrm{d} \sigma^{2}=\mathrm{d} x_{1}^{2}+\mathrm{d} x_{2}^{2}+2 \mathrm{~d} z^{2}
$$

and substitute (35) in (36), we get

$$
\begin{aligned}
\mathrm{d} S^{2}= & {\left[1+\frac{R^{\prime 2}(z) \bar{r}^{2}}{1-\kappa R^{2}(z) \bar{r}^{2}}\right] \mathrm{d} z^{2}+\frac{R^{\prime}(z) R(z) \bar{r} \mathrm{~d} z \mathrm{~d} \bar{r}}{1-\kappa R^{2}(z) \bar{r}^{2}} } \\
& +R^{2}(z)\left[\frac{\mathrm{d} \bar{r}^{2}}{1-\kappa R^{2}(z) \bar{r}^{2}}+\bar{r}^{2} \mathrm{~d} \theta^{2}\right]
\end{aligned}
$$


The form of (37) is similar to that of (12). The equations corresponding to (8) and (9) are

$$
\begin{gathered}
\mathrm{d} \sigma^{2}=R^{2}(z)\left(\frac{\mathrm{d} \bar{r}^{2}}{1-\kappa \bar{r}^{2}}+\bar{r}^{2} \mathrm{~d} \theta^{2}\right) \\
\mathrm{d} S^{2}=\mathrm{d} z^{2}+R^{2}(z)\left(\frac{\mathrm{d} \bar{r}^{2}}{1-\kappa \bar{r}^{2}}+\bar{r}^{2} \mathrm{~d} \theta^{2}\right)
\end{gathered}
$$

It is obvious that the curvature of (39) can not be constant $\kappa$ when $z \neq$ constant. In fact, by considering $r=R(z) \bar{r}$, the spherical surface equation (32) becomes

$$
R^{2}(z) \bar{r}^{2}+z^{2}=\frac{1}{\kappa}
$$

Because new variables are $\bar{r}$ and $z,(40)$ is not the equation of spherical surface and constant $\kappa$ is no longer curvature. When $z=Z_{0}=$ constant, according to (40), we have

$$
\bar{r}^{2}=\frac{1}{R^{2}\left(z_{0}\right)}\left(\frac{1}{\kappa}-z_{0}^{2}\right)=\frac{1}{K}
$$

Only in this case, constant $K$ represent the curvature of circle with radius $\bar{r}$ on the plane $z_{0}=$ constant. Similarly, by using $r=R(t) \bar{r}$ in supper spherical surface equation (4), we obtain

$$
R^{2}(t) \bar{r}^{2}-c^{2} t^{2}=\frac{1}{\kappa}
$$

Because new variables are $\bar{r}$ and $t,(42)$ is not the equation of three dimensional supper spherical surface and the constant $\kappa$ is no longer curvature again. When $t=t_{0}=$ constant, we have

$$
\bar{r}^{2}=\frac{1}{R^{2}\left(t_{0}\right)}\left(\frac{1}{\kappa}+c^{2} t_{0}^{2}\right)=\frac{1}{K}
$$

Only in this case, constant $K$ can be considered as the curvature of supper spherical surface with radius $\bar{r}$.

We calculate the curvature tensor of the metric (38). The non-zero metric tensors of (38) are

$$
\begin{array}{ll}
g_{11}=\frac{R^{2}}{1-\kappa \bar{r}^{2}} & g_{22}=R^{2} \bar{r}^{2} \\
g^{11}=\frac{1-\kappa \bar{r}^{2}}{R^{2}} & g_{22}=\frac{1}{R^{2} \bar{r}^{2}}
\end{array}
$$

The non-zero Christoffel signs are

$$
\Gamma_{11}^{1}=\frac{k \bar{r}}{1-\kappa \bar{r}^{2}} \quad \Gamma_{22}^{1}=-\left(1-\kappa \bar{r}^{2}\right) \bar{r} \quad \Gamma_{12}^{2}=\frac{1}{\bar{r}}
$$

According to (20), the only non-zero curvature tensor is

$$
R_{1212}=\frac{\kappa R^{2} \bar{r}^{2}}{1-\kappa \bar{r}^{2}}
$$

So according to the formula (16), the Riemannian curvature of (38) is

$$
K_{12}=\frac{R_{1212} p^{1} q^{2} p^{1} q^{2}}{\left(g_{11} g_{22}-g_{12} g_{12}\right) p^{1} q^{2} p^{1} q^{2}}=\frac{R_{1212}}{g_{11} g_{22}}=\frac{\kappa}{R^{2}}
$$

That is to say, the curvature of (38) is $\kappa / R^{2}(z)$, rather then $\kappa$. In fact when $R(z)=$ constant, let $\kappa \rightarrow \kappa R^{2}(z)$ in (38), we get (34).

Next, we calculate the curvature of (39). We consider $z$ as $x^{0}$. The metric tensors of (39) are

$$
\begin{array}{lll}
g_{00}=1 & g_{11}=\frac{R^{2}}{1-\kappa \bar{r}^{2}} & g_{22}=R^{2} \bar{r}^{2} \\
g^{00}=1 & g^{11}=\frac{1-\kappa \bar{r}^{2}}{R^{2}} & g_{22}=\frac{1}{R^{2} \bar{r}^{2}}
\end{array}
$$

The non-zero Christoffel signs are

$$
\begin{array}{ll}
\Gamma_{11}^{0}=-\frac{R R^{\prime}}{1-\kappa \bar{r}^{2}} \Gamma_{22}^{0}=-R R^{\prime} \bar{r}^{2} \quad \Gamma_{01}^{1}=\Gamma_{02}^{2}=\frac{R^{\prime}}{R} \\
\Gamma_{11}^{1}=\frac{k \bar{r}}{1-\kappa \bar{r}^{2}} \quad \Gamma_{22}^{1}=-\left(1-\kappa \bar{r}^{2}\right) \bar{r} \quad \Gamma_{12}^{2}=\frac{1}{\bar{r}}
\end{array}
$$

The non-zero curvature tensors are

$$
\begin{aligned}
& R_{0101}=-\frac{R R^{\prime \prime}}{1-\kappa \bar{r}^{2}} R_{0202}=-R R^{\prime \prime} \bar{r}^{2} \\
& R_{1212}=\frac{R^{2} \bar{r}^{2}\left(\kappa-R^{\prime 2}\right)}{1-\kappa \bar{r}^{2}}
\end{aligned}
$$

According to (16), we have

$$
\begin{aligned}
& K_{01}=\frac{R_{0101}}{g_{00} g_{11}}=-\frac{R^{\prime \prime}}{R} \quad K_{02}=\frac{R_{0202}}{g_{00} g_{22}}=-\frac{R^{\prime \prime}}{R} \\
& K_{12}=\frac{R_{1212}}{g_{11} g_{22}}=\frac{\kappa-R^{\prime 2}}{R^{2}}
\end{aligned}
$$

Because $K_{12}$ contains $R^{\prime 2}$, the result is different from (47). It means that the spatial curvatures of (38) and (39) are different. It implies that the dimensions of space can change curvature, despite that the metric form (39) of two dimensional curved surface is completely the same as (38). In fact, as we know that the metric $\mathrm{d} s^{2}=\mathrm{d} r^{2}+r^{2} \mathrm{~d} \theta^{2}+r^{2} \sin ^{2} \theta \mathrm{d} \phi^{2}$ is flat with zero curvature, but $\mathrm{d} \sigma^{2}=r^{2} \mathrm{~d} \theta^{2}+r^{2} \sin ^{2} \theta \mathrm{d} \phi^{2}$ is curved with curvature $\kappa=1 / r^{2}$. Related to the radius of spherical surface, the curvature is not a constant. 